\title{
Finalized configuration of magnetic shielding for LEReC Cooling Section
}

\author{
S. Seletskiy, V. De Monte, A. Di Lieto, A. Fedotov, \\ G. Mahler, G. McIntyre, J. Tuozzolo, D. Weiss
}

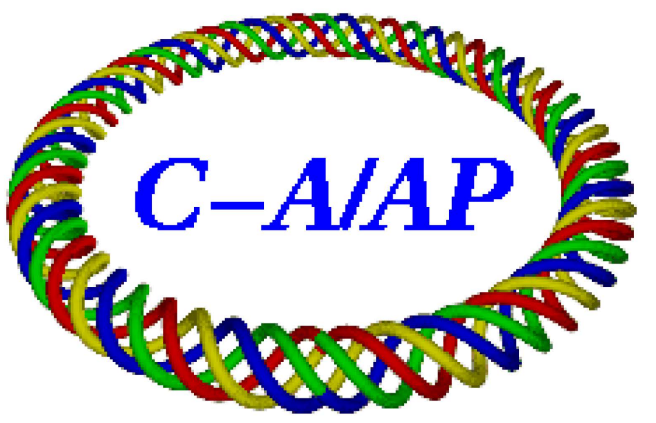

Collider-Accelerator Department Brookhaven National Laboratory Upton, N.Y. 11973

\section{U.S. Department of Energy Office of Science, Office of Nuclear Physics}

Notice: This document has been authorized by employees of Brookhaven Science Associates, LLC under Contract No. DE-SC0012704 with the U.S. Department of Energy. The United States Government retains a non- exclusive, paid-up, irrevocable, world-wide license to publish or reproduce the published form of this document, or allow others to do so, for United States Government purposes. 


\section{DISCLAIMER}

This report was prepared as an account of work sponsored by an agency of the United States Government. Neither the United States Government nor any agency thereof, nor any of their employees, nor any of their contractors, subcontractors, or their employees, makes any warranty, express or implied, or assumes any legal liability or responsibility for the accuracy, completeness, or any third party's use or the results of such use of any information, apparatus, product, or process disclosed, or represents that its use would not infringe privately owned rights. Reference herein to any specific commercial product, process, or service by trade name, trademark, manufacturer, or otherwise, does not necessarily constitute or imply its endorsement, recommendation, or favoring by the United States Government or any agency thereof or its contractors or subcontractors. The views and opinions of authors expressed herein do not necessarily state or reflect those of the United States Government or any agency thereof. 


\title{
Finalized configuration of magnetic shielding for LEReC Cooling Section
}

\author{
S. Seletskiy, V. De Monte, A. Di Lieto, A. Fedotov, G. Mahler, G. McIntyre, \\ J. Tuozzolo, D. Weiss
}

\section{Introduction}

In the LEReC Cooling Section (CS) the RHIC ions are traveling together with and getting cooled by the LEReC electrons. The required cooling rate [1] sets the limit of 150 urad on tolerable angles of the electrons in the CS. One of the components of overall electron angle is the angle of the e-beam trajectory with respect to the ion beam trajectory. We set $[2,3]$ the limit for electron trajectory angle to 100 urad.

It is critical [4,5] for preserving small trajectory angle to keep the transverse magnetic field inside the CS drifts within +/- $2.3 \mathrm{mG}[2-5]$.

The drifts in the CS must be shielded from the ambient magnetic fields of the RHIC tunnel, which can be as high as $0.5 \mathrm{G}$ [2], to minimize the transverse field inside the CS vacuum chamber.

In this paper we present the final design of the magnetic shielding of the LEReC CS and discuss the results of tests dedicated to studies of the shielding effectiveness.

\section{CS Shielding Design}

Each LEReC CS (Fig. 1) contains 8 solenoids combined with trajectory correctors and the BPMs located downstream of each solenoid. The distance between solenoid centers is $3 \mathrm{~m}$.

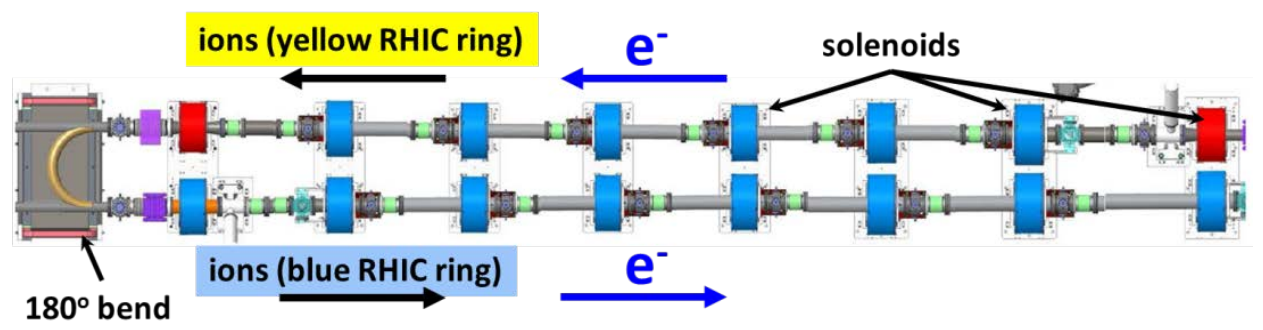

Figure 1: LEReC cooling sections.

The physical design of the shielding for each of the CS drifts was done in accordance to the analysis presented in [2,3]. The engineering design had to take into account the limitations produced by size of LEReC diagnostic equipment, by placement of solenoids electrical interface and by requirement to install the cooling section bake-out blankets. As a result, the typical CS drift is shielded by two cylindrical layers of $1 \mathrm{~mm}$ thick mu-metal (Fig. 2). The first layer is installed right on top of the 5" diameter vacuum pipe and the second layer has diameter of 14.52".

Each layer consists of 6 half cylinders, which are designed in such a way that when installed both horizontal and vertical seams have 1.5 " overlap of mu-metal. 


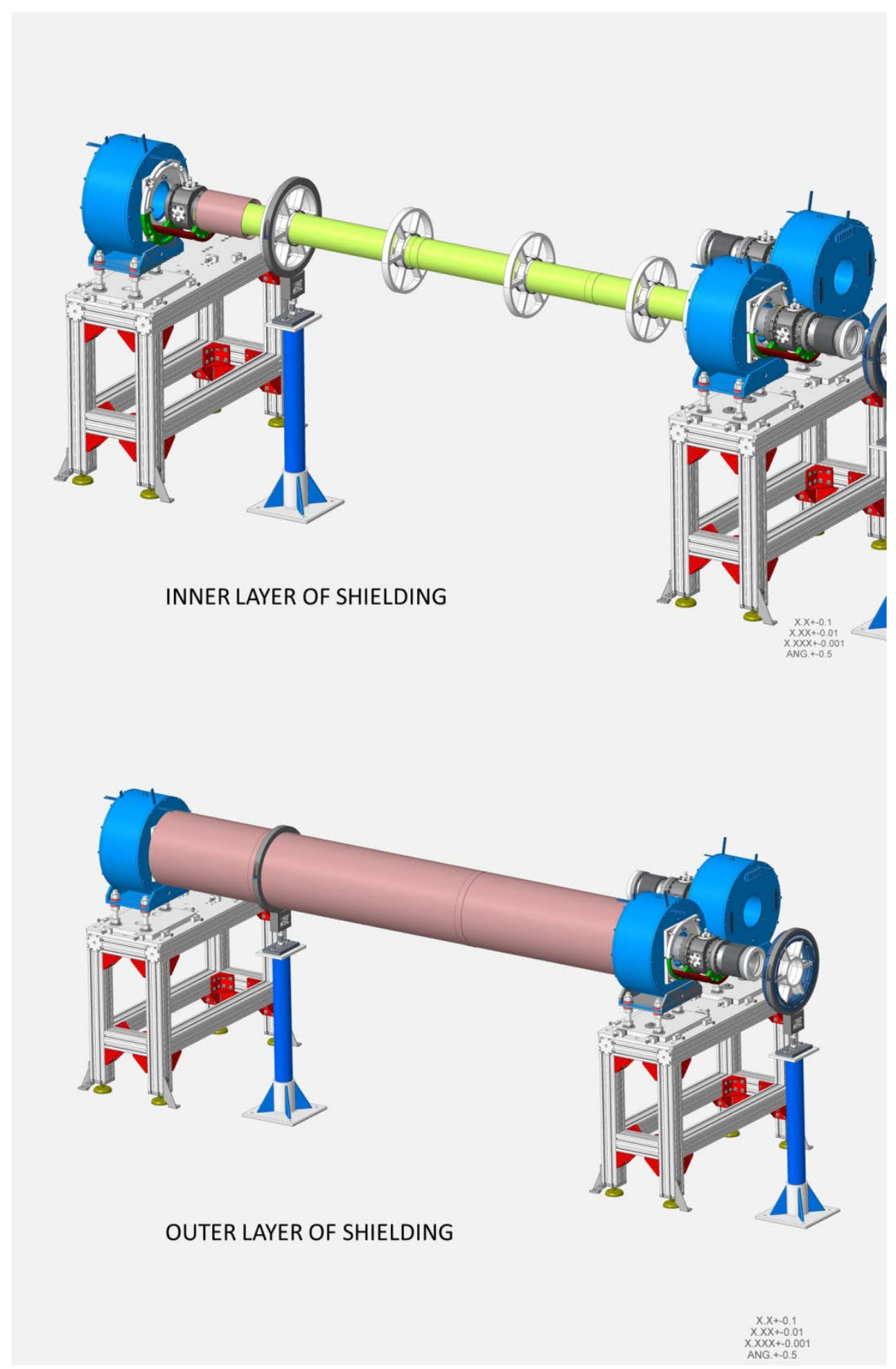

Figure 2: Two layers of CS shielding.

The shielding was produced by Ad-Vance Magnetics [6]. They measured the magnetic permeability to be 11500 at low frequency applied field of $18 \mathrm{G}$. Assuming that at constant sub-Gauss fields $\mu \approx 11000$ we expect $[2,3]$ that for one layer the attenuation factor will be:

$$
S=1+\mu \frac{d}{D}=87
$$

and for two layers the attenuation factor will become: 


$$
S=S_{1} S_{2}\left(1-\left(\frac{D_{1}}{D_{2}}\right)^{2}\right)+S_{1}+S_{2}+1=2500
$$

\section{Shielding Test}

\section{Test Setup}

To test the shielding we built a mockup of the LEReC CS part consisting of two solenoids and $3 \mathrm{~m}$ drift. We installed the shielding on this test bench and performed multiple measurements of obtained attenuation factor. We checked the effectiveness of the shielding with solenoids being powered on and off. We also tested how the shielding withstands the bake-out of the vacuum chamber.

The test bench was installed not inside the RHIC tunnel but in a separate building. The ambient fields in this building were similar to the fields we expect to have in the tunnel. Therefore, the obtained results do not give us the exact fields inside the CS drifts but rather provide us with the remnant field levels that we can reasonably expect in our Cooling Section.

The measurements were performed with Magnetoresistive Milligauss Meter [7], which has a guaranteed accuracy of $0.5 \mathrm{mG}$ and a precision of $0.01 \mathrm{mG}$.

The Gauss-meter contains three directional magnetic gauges mounted off-center in a 3D probe ( $25 \mathrm{x}$ $25 \times 38 \mathrm{~mm}$ rectangular block). Each gauge is mounted about $4 \mathrm{~mm}$ under the surface of the probe with +/- $2 \mathrm{~mm}$ precision. The probe was installed inside the cart that was dragged through the vacuum chamber with $5 \mathrm{~cm}$ steps. Since the cart was dragged by hand the cart position repeatability was a few millimeters. The center of the cart was centered with the centerline of the vacuum tube with a few millimeter precision. The cart bottom was biased with some weight to minimize cart's rotation around the longitudinal axis.

\section{Test Timeline}

Multiple shielding tests were performed on the test bench.

Due to the schedule pressure, we started with taking the baseline measurement without the shielding and then immediately switching to the measurement with both layers installed. These initial measurements were done with the solenoids turned off.

Next, we performed the measurement with solenoids powered to the maximum current and then with the solenoids turned off again.

Later, the test of how the shields withstand the vacuum chamber bake-out was performed.

After one of the solenoids was removed (due to the requirements of the LEReC installation schedule), we repeated tests with remaining solenoid on and off. This time the solenoid was cycled 10 times.

Finally, the shielding measurement with single layer was performed.

\section{Test Results: Attenuation Factors}

The plots of magnetic field inside the vacuum chamber for 2 layers and 1 layer of shielding as well as measurements with no shielding are presented in Fig. 3. All three measurements were performed with solenoids turned off. The measurements with 2 and 1 layers of shielding were performed with only one solenoid left on the test bench and after this solenoid was cycled multiple times. 


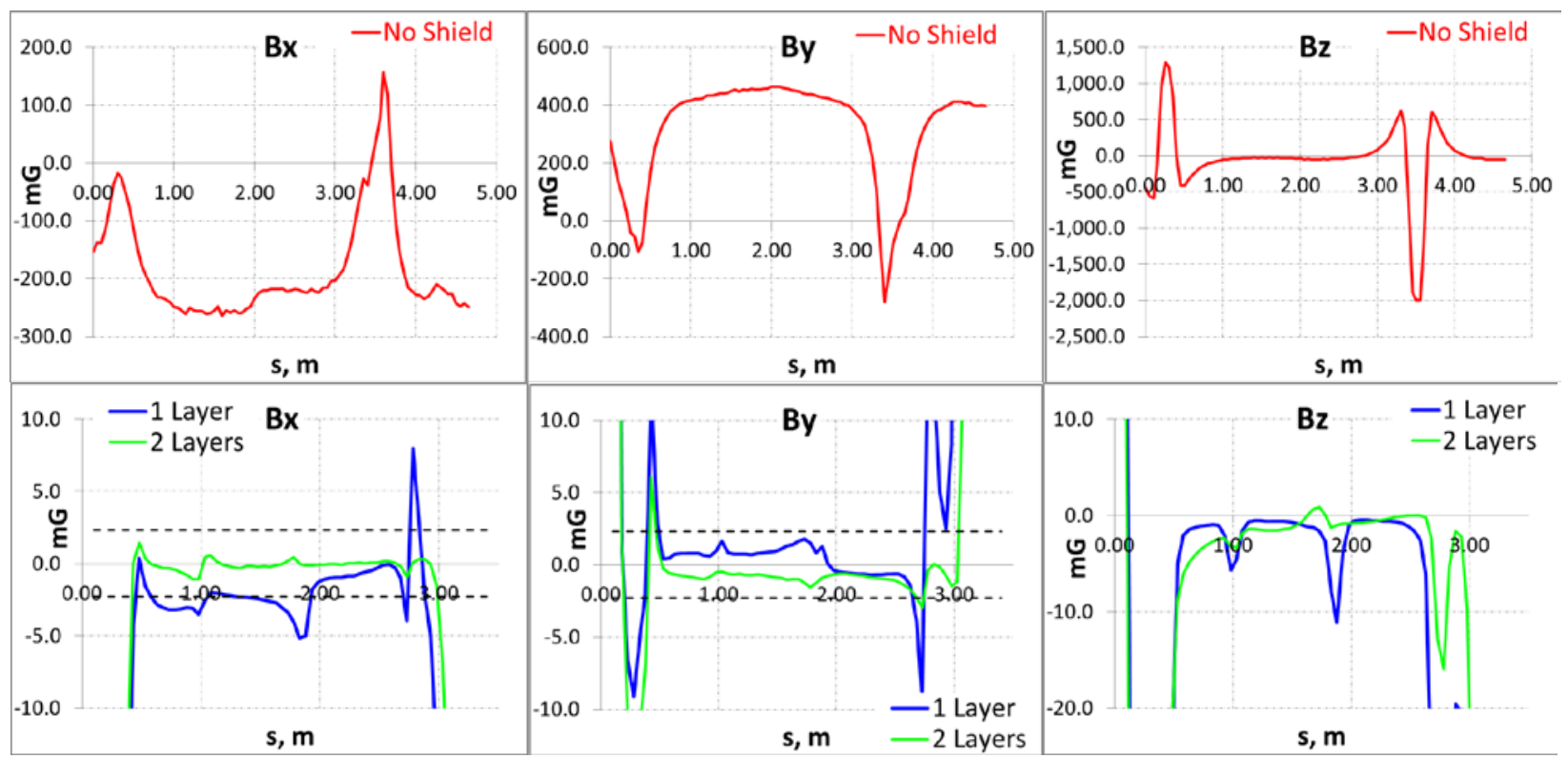

Figure 3: Horizontal (left), vertical (center) and longitudinal (right) magnetic fields measured without shielding (upper plots) and with 1 (lower plots, blue line) and 2 (lower plots, green line) layers of shielding. The plots for shielded fields are zoomed on the shielded area to show the field quality in details. The dotted black lines represent $+/-2.3 \mathrm{mG}$ field levels.

The spikes in longitudinal field for the shielded measurements correspond to the vertical seams between shielding cylinders. Although there is $1.5^{\prime \prime}$ overlap of mu-metal at the seams it is apparent that the flux concentrated in the shields leaks inside the vacuum chamber at seams locations.

The respective spikes of the transverse field are related to the fact that the transverse gauges are not centered with respect to the shielding. As a matter of fact, by comparing longitudinal and transverse field plots (Fig 4) for one of the measurements and estimating the gauges displacements from the chamber center from:

$$
B_{\perp}=-\frac{r}{2} \frac{d B_{z}}{d z}
$$

we obtain $\mathrm{x}$-displacement of $3 \mathrm{~cm}$ and $\mathrm{y}$-displacement of $-1 \mathrm{~cm}$, which is reasonable for the probe setup utilized in the measurement.

The well-shielded area covers $2.5 \mathrm{~m}$ of the drift between solenoids (from $\mathrm{z}=0.5 \mathrm{~m}$ to $\mathrm{z}=3 \mathrm{~m}$ ). The effective average attenuation factor in this area is $S_{x} \approx 1300$ and $S_{y} \approx 900$ for two layers of shielding. This is about factor of 2 - 2.5 smaller then theoretical prediction for $\mathrm{S}$.

We attribute this discrepancy to several factors.

First of all, our shielding does not consist of ideal infinitely long cylinders. The cylinders have finite length and each of them is built from six pieces.

Second, the magnetic permeability of the material for sub-Gauss DC fields might be smaller than the permeability measured for $18 \mathrm{G}$ AC field of low frequency.

In any case, as we will show below, the obtained attenuation of the ambient magnetic field is adequate for the purpose of shielding of the LEReC Cooling Section. 

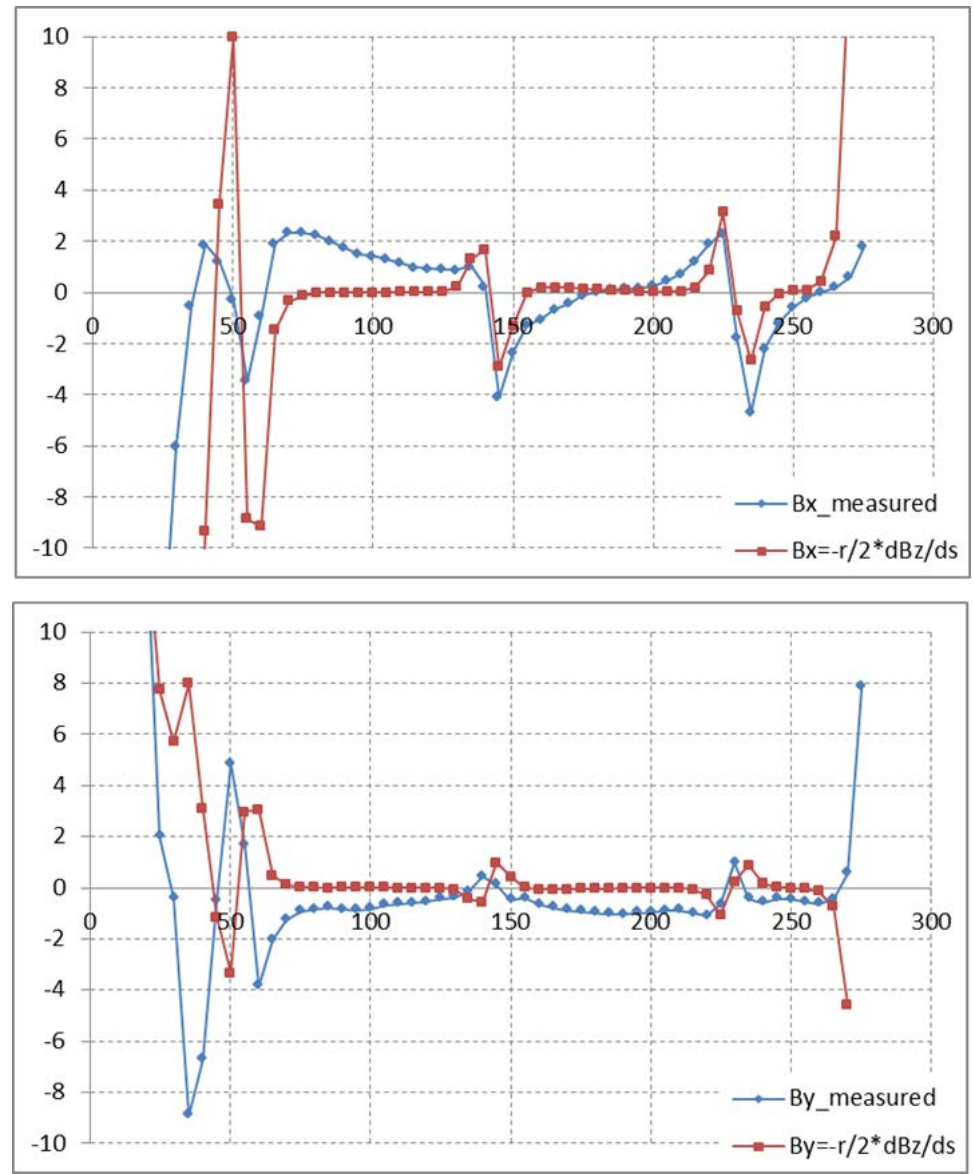

Figure 4: Transverse fields (horizontal - upper plot, and vertical - lower plot) inside the shielded area. Blue line corresponds to the measured field, the dark red line is the field calculated from the measured longitudinal field under assumption of $d x=3 \mathrm{~cm}$ and $d y=-1 \mathrm{~cm}$.

\section{Test Results: Exposure to Bake-out}

We performed a dedicated test to test the feasibility of leaving the shielding in place when the vacuum chamber requires a bake-out.

The outer shielding was removed. The baking blankets were wrapped around the first shielding layer. The vacuum chamber with the first layer in place was baked to $250 \mathrm{C}$. After the baking the second layer was reinstalled.

By comparing the measurements before and after the baking (Fig. 5) we concluded that the exposure of the mu-metal to the bake-out temperatures does not cause the deterioration of its shielding quality.

We attribute the small improvement in the measured "post-baked" fields to the better job that was done on re-installation of the second layer. 


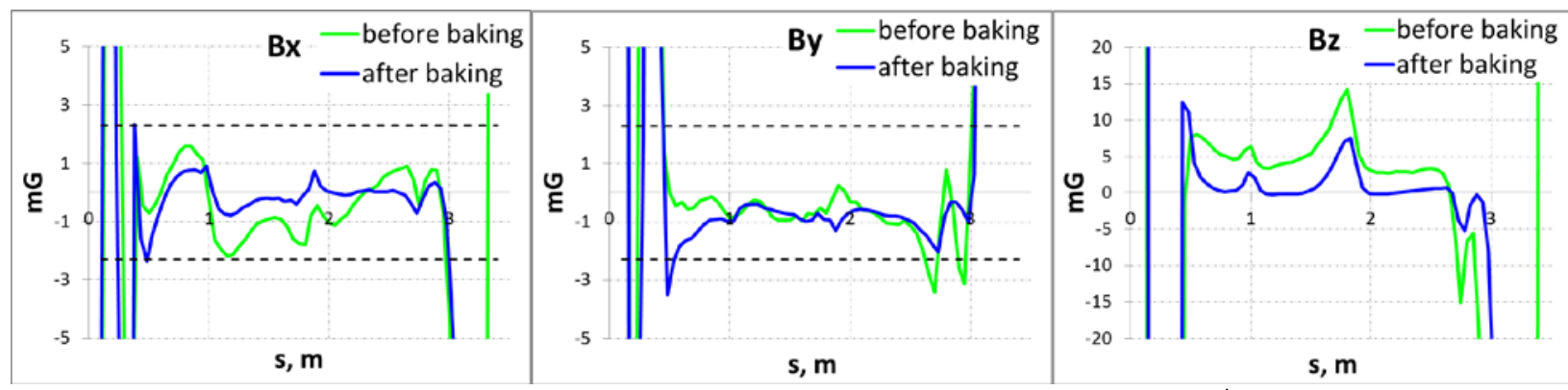

Figure 5: Magnetic fields measured before and after the baking of the $1^{\text {st }}$ shielding layer.

\section{Test results: Shielding in Presence of Solenoidal Field}

We performed the measurement of the field inside the shielded area with the solenoids turned on up to the maximum current.

With solenoids powered the additional field flux is partially captured by the shielding and is "leaking" inside the vacuum chamber through the vertical seams.

Another important effect is the changed remnant magnetization of the solenoids after the solenoids are turned off. Due to this effect the field inside the shielded area changes as the solenoids are cycled. The fields for both solenoids on and off are stabilizing after several cycles.

Figure 6 shows measured fields after 10 solenoid cycles for both solenoids turned on and off. As we will show the measured fields are adequate for preserving the good quality of electron beam.
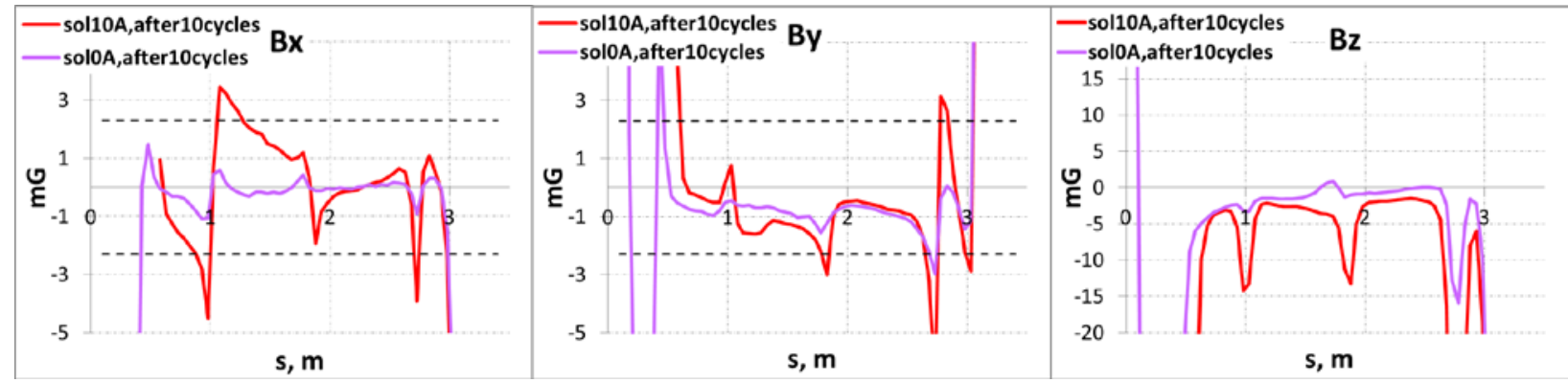

Figure 6: Fields measured for solenoids turned on and off (after 10 solenoid cycles).

\section{Analysis}

\section{Implications for trajectory alignment}

Let us assume that the remnant field inside the vacuum chamber is the only source of e-beam trajectory angles inside the cooling section. That is, we are assuming that the CS solenoids and BPMs are perfectly aligned and we are ignoring the effect of ion-electron kick.

First, we simulated the e-beam trajectory in the field measured for the turned-off solenoid. We simulated both uncorrected and corrected trajectories. The corrected trajectory is obtained by applying such an instantaneous kick at the starting point of the simulation that the trajectory displacement "measured" at simulation exit point is zero in both horizontal $(x)$ and vertical ( $y$ ) directions.

Next, we simulated the e-beam trajectory for the fields measured for the turned-on solenoid. Again, both the uncorrected and corrected trajectories were simulated. 
All simulations were done for $1.6 \mathrm{MeV}$ beam. The simulations were performed by numerically solving (with explicit Euler method) equations:

$$
\left\{\begin{array}{l}
\frac{d x}{d z}=\theta_{x} \\
\frac{d \theta_{x}}{d z}=\frac{B_{y}}{B \rho}
\end{array} ; \quad\left\{\begin{array}{l}
\frac{d y}{d z}=\theta_{y} \\
\frac{d \theta_{y}}{d z}=\frac{B_{x}}{B \rho}
\end{array}\right.\right.
$$

The magnetometer doesn't respond to the fields higher than $2 \mathrm{G}$ and shall be kept out of high field region. Therefore, for the turned on solenoids we could simulate beam trajectory only inside $2.75 \mathrm{~m}$ long drift.

In our simulations we ignored the presence of longitudinal field in the CS drifts since it is too small to cause non-negligible $x-y$ coupling. We used the raw measured transverse fields not corrected for the probe displacement from the chamber axis.

Figures 7 and 8 show the results of simulations of corrected trajectories with the solenoid off and on respectively.

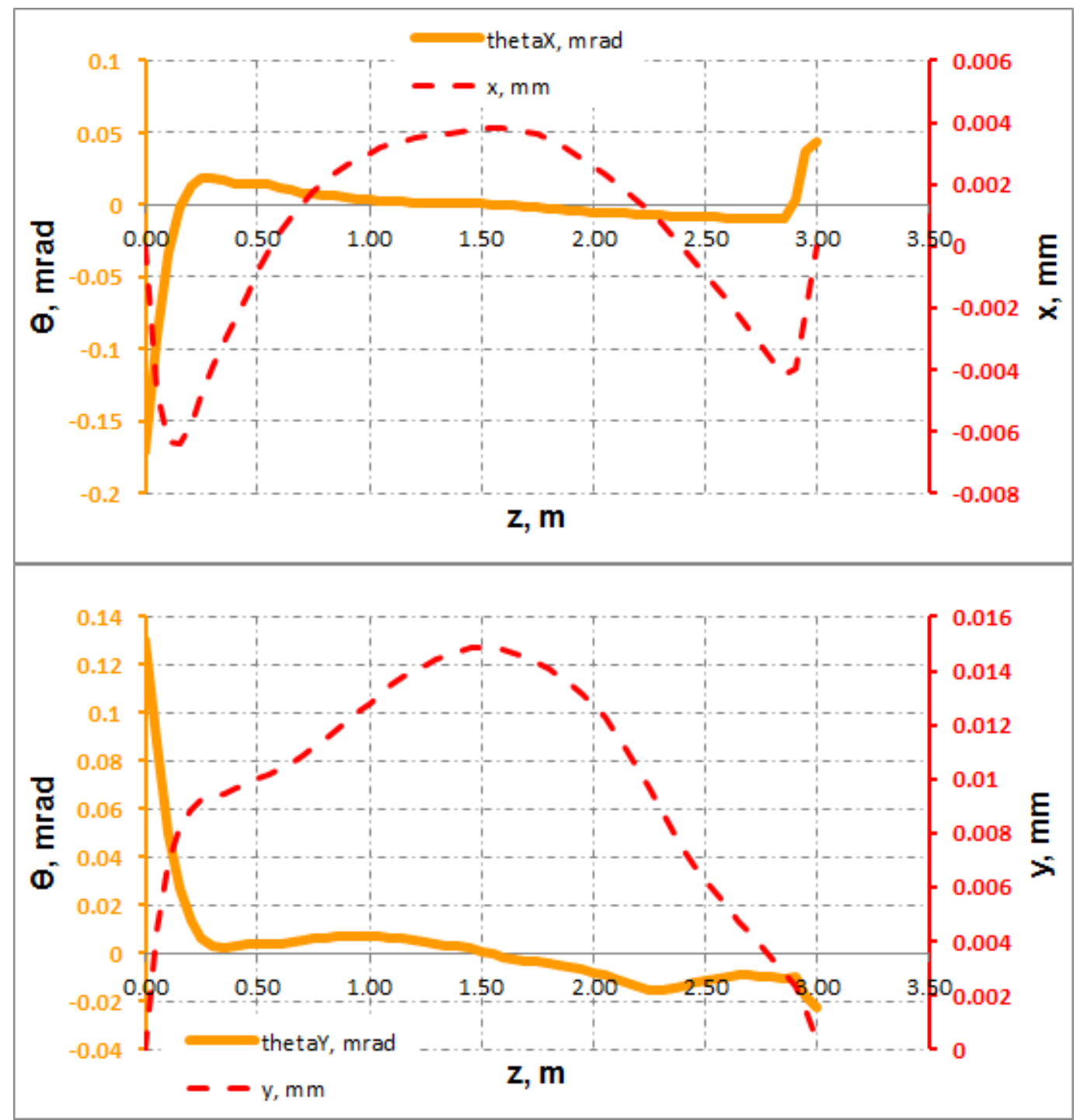

Figure 7: Corrected horizontal (upper plot) and vertical (lower plot) electron beam trajectories (dotted red lines) and trajectory angles (solid orange lines) for the case of turned off solenoids. 


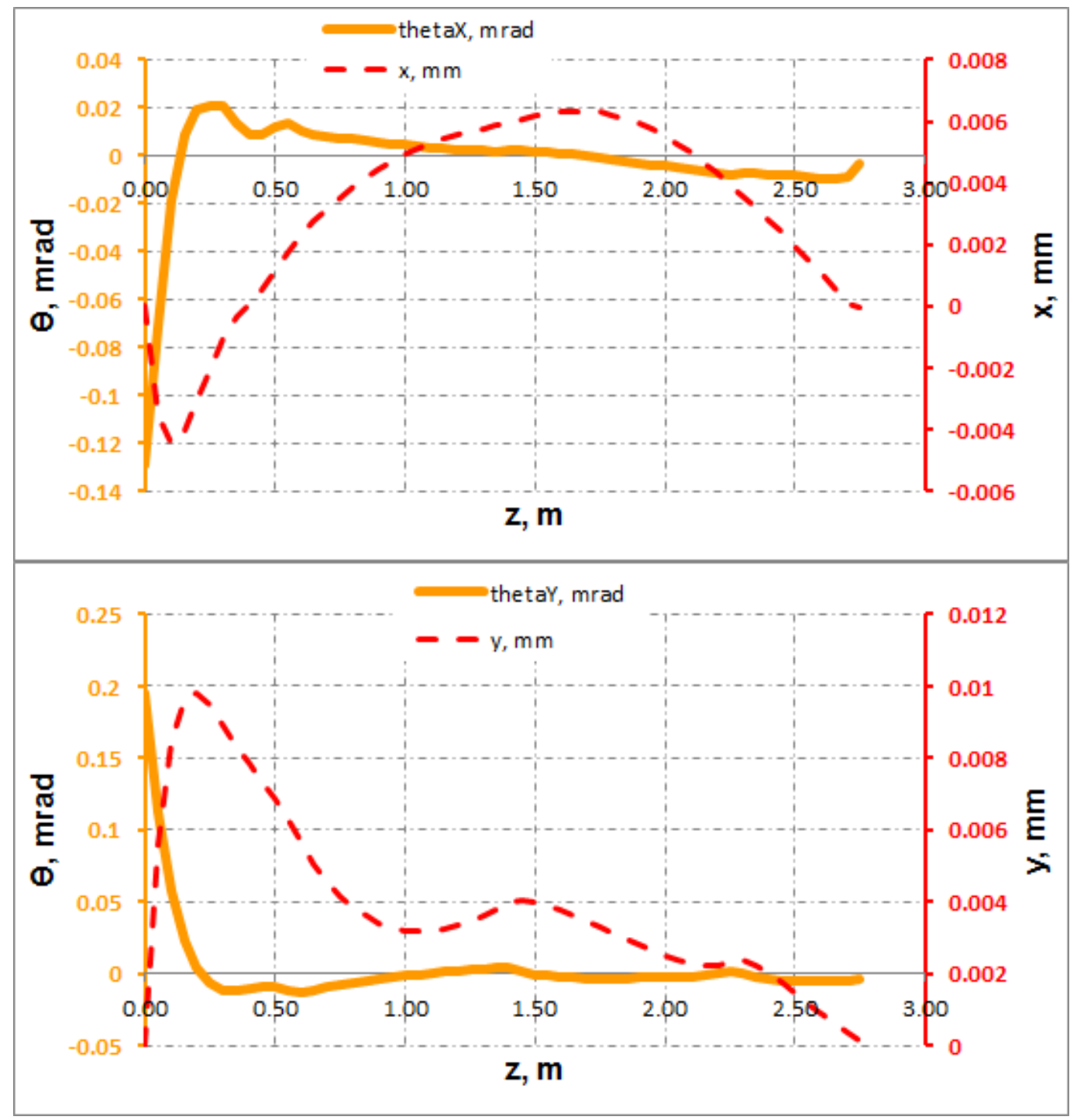

Figure 8: Corrected horizontal (upper plot) and vertical (lower plot) electron beam trajectories (dotted red lines) and trajectory angles (solid orange lines) for the case of turned on solenoids.

As one can see, the obtained angles in shielded area of the LEReC CS drift are quite satisfactory for the electron cooling.

\section{Implications for CS solenoids alignment}

The detailed procedure for CS solenoid alignment is described in [4]. In a nutshell, after the CS BPMs are aligned to the ideal e-trajectory provided by the ion beam each CS solenoid is individually turned on and we observe the response of downstream BPMs to the changes in the current of particular solenoid.

This alignment algorithm relies on the assumptions of negligibly small fields in solenoid to solenoid drifts, which are not changing with solenoid current. Since our measurements show that the field in the shielded drift does depend on the solenoid current one must study the implications of such an effect on the CS solenoid alignment procedure.

We do not have a measurement of the drift fields for two-solenoid assembly with only one solenoid turned on. So, instead, we will use the field map with 2 solenoids on, which is shorter than the map with 
solenoids off (see, previous section for explanations), and continue with the "solenoids off" map on one side of the map to simulate the beam trajectory one can expect to have in CS alignment procedure.

We start with corrected trajectories found for the turned-off solenoids demonstrated in Fig. 7. The trajectories were obtained with horizontal and vertical correctors set to $130 \mathrm{urad}$ and -170 urad respectively.

Next, keeping the correctors values, we "turn on" one solenoid and simulate the new beam trajectory in the filed map constructed as described above. Figure 9 shows the result of such simulations.

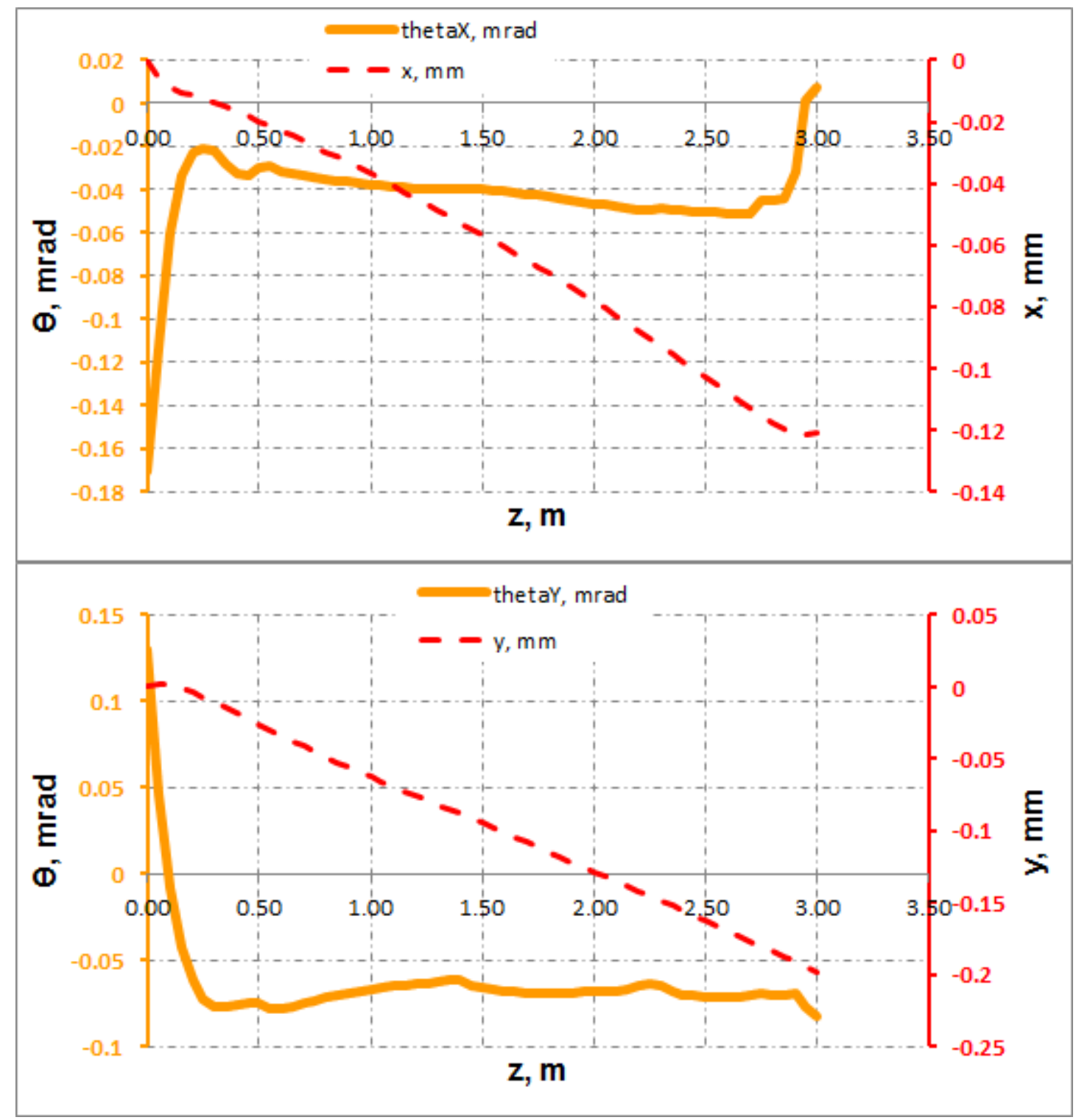

Figure 9: Horizontal (upper plot) and vertical (lower plot) beam trajectories for one turned on solenoid.

As one can see the expected beam displacement at downstream BPM due to changed transverse field in the drift is $200 \mathrm{um}$ at maximum. Such displacement corresponds to $0.15 \mathrm{~mm}$ error in determined solenoidal shift and 200 urad error in determined solenoidal inclination (see [4] for details on how these numbers must be computed). Such errors in solenoid positioning are smaller than $0.5 \mathrm{~mm}$ and $0.5 \mathrm{mrad}$ positioning requirements derived in [4]. 
It is also important to notice that in our analyses we on purpose overestimated the effect of field change with solenoid current change. We assumed that the field map is changing at the center of the vacuum chamber while in reality a lot of this change is caused by off-center positioning of the magnetic probe. Or, in other words, we assumed that on the test bench the solenoid is not misaligned with respect to the "trajectory" probed by the magnetometer, while we know for the fact that there is a few centimeter misalignment caused by the constructional properties of the probe.

\section{Conclusion}

We described the final design of the magnetic shielding for the LEReC cooling section.

The measurements of remnant magnetic field inside the shielded drift between two solenoidal modules were performed.

The analysis of the measured field map shows that the designed shielding satisfies the requirements of the LEReC cooling section.

\section{References}

[1] A. Fedotov et al., WEA4CO05, NAPAC16, 2016.

[2] S. Seletskiy et al., C-A/AP/561, BNL-112084-2016-IR, 2016.

[3] S. Seletskiy et al., WEPOB61, NAPAC16, 2016.

[4] S. Seletskiy et al., BNL-112720-2016-IR, 2016.

[5] S. Seletskiy et al., TUPAB145, IPAC17, 2017.

[6] www.advancemag.com

[7] http://www.trifield.com/ 